\title{
Natura@economía
}

ISSN 2226-9479 (Versión electrónica) Website: http://revistas.lamolina.edu.pe/index.php/neu

\section{Evaluando el desarrollo sostenible en la Región Junín}

\author{
Assessing sustainable development in the Junín Region \\ Carlos Enrique Orihuela Romero ${ }^{1 *}$; Cindy Silva Vivanco ${ }^{2}$
}

\begin{abstract}
${ }^{1}$ Facultad de Economía, Universidad Nacional Agraria La Molina, Lima, Perú. Email: corihuela@lamolina.edu.pe
${ }^{2}$ Facultad de Economía, Universidad Nacional Agraria La Molina, Lima, Perú. Email:cindysv50@gmail.com
\end{abstract}

Recepción: 25/09/2019; Aceptación: 15/12/2019

\begin{abstract}
Resumen
El objetivo del estudio es evaluar la sostenibilidad de la economía del departamento Junín durante el periodo 2005-2011. Para ello se utilizó el criterio Inversión Genuina, bajo el cual si el valor de la base productiva ha sido no decreciente en un periodo dado (inversión genuina positiva), entonces la economía ha estado en esa senda durante tal periodo. Los resultados sugieren que Junín estuvo en esa senda durante el periodo en análisis. Si bien la economía departamental presenta condiciones favorables para su futuro crecimiento, algunos capitales -como el institucional- deben articularse adecuadamente con el resto de la base productiva a fin de que la economía local pueda mantenerse en la senda del desarrollo sostenible.
\end{abstract}

Palabras clave: Desarrollo sostenible; inversión genuina; Junín; riqueza.

\begin{abstract}
The objective of the study is to evaluate the sustainability of the economy of the Junín department during the period 2005-2011. For this, the Genuine Investment criterion was used, under which if the value of the productive base has been non-decreasing in a given period (positive genuine investment), then the economy has been on that path during that period. The results suggest that Junín was on that path during the period under analysis. Although the departmental economy presents favorable conditions for its future growth, some capitals - such as the institutional one - must be properly articulated with the rest of the productive base so that the local economy can stay on the path of sustainable development.
\end{abstract}

Key words: Sustainable development; genuine investment; Junin; wealth.

Forma de citar el artículo: Orihuela, C.; Silva, C. 2019. Evaluando el desarrollo sostenible en la Región Junín. Natura@economía 4(2): 77- 93 (2019). 


\section{Introducción}

A raíz de la reciente coyuntura mundial de altos precios del petróleo y algunos metales (a partir del año 2005), el Perú inició un proceso de crecimiento económico nunca antes experimentado, lográndose incluso tasas de crecimiento anuales superiores al 7\%. La tasa de crecimiento del Valor Agregado Bruto durante (VAB) los periodos 2005/2006, 2006/2007, 2007/2008, $2008 / 2009,2009 / 2010$ y 2010/2011 fueron: 6,$4 ; 7,6 ; 8,5 ;-0,2 ; 7,6$ y 5,7 ; respectivamente (INEI, 2013 ${ }^{\mathrm{a}}$ ). Esto ha conllevado a una mayor dinámica en la economía local. Tal fue este crecimiento que incluso ante la crisis financiera del año 2008, la rápida mejora en los precios de exportación, aunada a las fortalezas de la economía peruana y las acertadas políticas económicas implementadas, logró que la velocidad de recuperación de la economía peruana fuera más rápida que la de sus pares de América Latina (MEF, 2011).

Sin embargo, el desempeño económico no ha sido homogéneo, puesto que ha diferido al interior del país y estas diferencias van más allá de la clasificación geográfica. Algunas regiones lograron crecer rápidamente, mientras que otras, quedaron rezagadas respecto al crecimiento departamental promedio. En particular, algunos departamentos pasaron a ser "emergentes" debido al desarrollo de la agroindustria y la minería, entre otros factores. En otros departamentos, el desarrollo de la industria del turismo contribuyó al dinamismo económico. En ambos casos, el empleo aumentó rápidamente. Sin embargo, en la gran mayoría de las regiones mineras del Perú ha sido difícil iniciar procesos de industrialización (Armendáriz et al., 2011).

Durante este periodo, los departamentos típicamente mineros como Cajamarca y Junín presentaron niveles de ingreso per-cápita y tasas de crecimiento anual del ingreso per-cápita por debajo del promedio nacional. Los ingresos per-cápita de estos departamentos -como promedio del periodo 2005-2011- oscilaron entre 1,752 y 3,156 soles (constantes 1994) cuando el promedio nacional fue 6,442 (INEI, 2012a). Esto corrobora el hallazgo de Armendáriz et al. (2011) en el sentido que algunos departamentos, a pesar de su riqueza, han presentado niveles rezagados de crecimiento, incluso en comparación al promedio nacional.

Si bien la tasa de crecimiento del PIB per-cápita es vista como un indicador del crecimiento económico, no necesariamente es la mejor para evaluar el desempeño económico de una economía. Cuando una economía extrae sus recursos naturales no renovables (por ejemplo, metales), eventualmente incrementa su dinámica económica y su PIB, pero al mismo tiempo reduce su stock de capital natural, y mermando eventualmente su capacidad para generar ingresos (provenientes del capital natural) en el futuro.

En consecuencia, una economía puede tener un PIB creciente durante un corto periodo y luego presentar niveles decrecientes. Esto es un problema para las economías altamente dependientes de sus recursos naturales, especialmente en recursos no renovables como los metales. Este es el caso de varios departamentos en el Perú.

Así, departamentos que poseen un PIB per-cápita por encima del promedio nacional e incluso con elevadas tasas de crecimiento no garantizan mantener un nivel de bienestar humano no decreciente en el tiempo. Lo contrario también es válido: departamentos que han presentado débiles niveles de PIB per-cápita bien podrían estarse preparando para satisfacer niveles crecientes de bienestar en el futuro.

Por ende, el PIB no permite evaluar apropiadamente si el bienestar humano vinculado a la actividad económica puede ser al menos no decreciente en el tiempo. El problema de las tradicionales medidas de ingreso es que no permiten inferir mucho sobre el bienestar humano futuro. Simplemente constituyen la imagen de un momento particular de la economía. Esto ya fue señalado por Dasgupta y Mäler (2000), en el sentido que el PIB (en un sentido estricto, el PNN) no es el más apropiado para las tareas que normalmente se le asignan.

Dasgupta y Mäler (2001) propusieron el indicador Inversión Genuina como el más apropiado para evaluar si una economía ha estado en la senda del desarrollo sostenible durante un periodo dado. Este indicador económico consiste en medir el cambio del valor de la base productiva o riqueza de 
una economía durante un periodo. Si una economía acumula riqueza entonces tendrá la capacidad para enfrentar o satisfacer las necesidades futuras, de manera que la economía en análisis estaría en capacidad de alcanzar su nivel de desarrollo deseado. Esto supera el problema del PIB per-cápita.

Evaluar el desarrollo sostenible es fundamental para las economías que extraen recursos naturales, cuyos niveles de extracción y bajos niveles de reinversión de rentas pueden hacer peligrar la satisfacción de necesidades de las generaciones futuras. En otras palabras, el bienestar de una economía depende de la venta de estos recursos y no se sabe si se está compensando adecuadamente el desgaste del capital de manera que sea posible mantener las posibilidades intactas de desarrollo para el futuro.

La hipótesis de este estudio es determinar si un departamento con bajos niveles de PIB per-cápita pero rico en recursos naturales puede mantener sus posibilidades de desarrollo en el futuro. Para esto caso se elige al departamento Junín debido a que: i) es uno de los departamentos mineros con niveles de PIB per-cápita por debajo del promedio nacional, y ii) dispone de mayor información económica con respecto a otros departamentos.

Por ello, el objetivo es calcular la inversión genuina para el departamento de Junín durante el periodo 2005-2011 utilizando el indicador Inversión Genuina, el cual goza de consenso de la literatura económica para evaluar el desarrollo sostenible de las economías, utilizándose una variante por el World Bank (2006; 2011) para determinar la sostenibilidad de las economías de sus países miembros. El stock de capital minero es -a priori- uno de los más importantes en el stock de capital total de Junín. La información física de este stock (reservas probadas) solo está disponible a partir del año 2005 hacia adelante. Por este motivo, se eligió ese periodo de estudio, aun cuando un análisis de sostenibilidad debería ser efectuado idealmente para un periodo más prolongado.

Dasgupta y Mäler (2001) proponen el indicador Inversión Genuina (IG) como el más apropiado para evaluar el desarrollo sostenible de una economía. Ellos sostienen que una senda de desarrollo es sostenible si el bienestar social es no decreciente en toda la senda. El modelo parte de la función de bienestar social (V), la cual se define como el valor presente de la utilidad (agregada o social) a lo largo de la senda. Así, V equivale a un bienestar intergeneracional. Koopmans (1960; 1965) demostró que, bajo condiciones generales, $\mathrm{V}$ debería ser entendido como:

$\mathrm{V}_{\mathrm{t}}=\sum_{\mathrm{s}=\mathrm{t}}^{\infty} \mathrm{Us} /(1+\delta)^{\mathrm{s}-\mathrm{t}}$

donde $\delta$ es la tasa de descuento de la utilidad. Como $U$ no es observable, Samuelson (1961) propuso que la riqueza actual (W) debería ser igual al valor presente del consumo futuro (2): lo que se consumirá en el futuro dependerá del nivel de riqueza actual. En otras palabras, la riqueza actual de una economía es igual al flujo descontado de los niveles que consumo que alcanzará en el futuro.

$\mathrm{V}_{\mathrm{t}} \approx \mathrm{V}_{\mathrm{t}}=\sum_{\mathrm{s}=\mathrm{t}}^{\infty} \mathrm{U}(\mathrm{Cs}) /(1+\delta)^{\mathrm{s}-\mathrm{t}}$

siendo $\mathrm{C}_{\mathrm{s}}$ el consumo del periodo $\mathrm{s}$ y $\mathrm{U}(\mathrm{C})$ la utilidad de ese consumo, la cual contiene todo lo que afecta al bienestar en un periodo dado. Con esta predicción es factible pronosticar el bienestar social:

$\mathrm{V}_{\mathrm{t}}=\sum_{\mathrm{t}}^{\infty} \mathrm{U}\left(\alpha\left(\mathrm{s}, \mathrm{t}, \mathrm{K}_{\mathrm{t}}\right)\right) /(1+\delta)^{\mathrm{s}-\mathrm{t}}$

Obviamente, $\mathrm{V}$ es una función del stock de capital inicial y de su mecanismo de asignación. El patrón de consumo (y el bienestar intertemporal) depende de la evolución de la base productiva de la economía, de manera que en algún momento la producción generada por esta base es asignada entre consumo e inversión en diferentes formas de capital.

Tal asignación está determinada por dos tipos de mecanismos: autónomo y no-autónomo. Una pequeña economía abierta generalmente tiene un mecanismo de asignación no-autónomo ya que sus decisiones dependen también de factores 
externos. Asumiendo un mecanismo de asignación no-autónomo, $\mathrm{V}$ será una función explicita del tiempo y para el caso de un solo tipo de capital, $\mathrm{K}$ :

$\mathrm{V}_{\mathrm{t}}=\mathrm{V}(\mathrm{K}(\mathrm{t}), \mathrm{t})$

Calculando el diferencial total de (4):

$\mathrm{dVt}=(\partial \mathrm{V} / \partial \mathrm{K}) \mathrm{dK}+(\partial \mathrm{V} / \partial \mathrm{t}) \mathrm{dt}$

Aplicando la derivada total con respecto a t, (5) puede ser re-expresado como:

$\mathrm{dV} / \mathrm{dt}=\mathrm{p}_{\mathrm{t}}(\mathrm{dK} / \mathrm{dt})+(\partial \mathrm{V} / \partial \mathrm{t})$

Donde $p_{t}=\partial \mathrm{V} / \partial \mathrm{K}$. El miembro del lado izquierdo de la expresión (6) equivale a la inversión genuina $(\mathrm{I}=\mathrm{dV} / \mathrm{dt})$, la cual se define como la variación del bienestar intertemporal, variación de la riqueza o cambio en la base productiva. Si esta es no decreciente en el tiempo entonces la generación futura tendría al menos las mismas oportunidades para generar bienestar tal como las tuvo la generación predecesora.

De esta forma, si $\mathrm{I}_{t}>0$ entonces la riqueza aumentó en el periodo t. En otras palabras, la economía se encontró en la senda sostenible durante el periodo señalado, puesto que generó los recursos (en valor social) necesarios para satisfacer el desarrollo futuro. Caso contrario, la economía no se encontró en la senda sostenible en el periodo t. Esto es una señal de una potencial caída en el bienestar humano futuro.

El primer miembro del lado derecho de la expresión (6) representa la variación del capital $\mathrm{K}$ valorado por su respectivo precio cuenta constante, $\mathrm{p}_{\mathrm{t}}$. Este precio se define como el valor presente de los futuros beneficios netos de un capital resultante de una perturbación en su stock inicial o como la contribución de una unidad adicional de capital al bienestar social (7). Operacionalmente, la correcta estimación de este precio cuenta es clave (UNU-IHDP y UNEP, 2012).

$\mathrm{p}_{\mathrm{t}}=\partial \mathrm{Vt} / \partial \mathrm{Kt}=\sum \frac{\left[\frac{\partial \mathrm{U}\left(\alpha\left(\mathrm{s}, \mathrm{t}, \mathbf{K}_{\mathrm{t}}\right)\right)}{(1+\delta)^{-\mathrm{t}}}\right]}{(1+\delta)^{\mathrm{st}}}$
El segundo miembro $(\partial \mathrm{V} / \partial \mathrm{t})$ es conocido como el "drift term" el cual es independiente de la base productiva y representa el efecto de cambios exógenos tales como variaciones tecnológicas e institucionales, productividad y bienes públicos globales.

Extendiendo (7) en tiempo discreto y para el caso de tres tipos de capital: artificial $(\mathrm{K})$, natural $(\mathrm{N})$ y humano $(\mathrm{H})$ se obtiene $(8)$ :

$\mathrm{I}_{\mathrm{t}}=\Delta \mathrm{W}=\sum_{\mathrm{i}}\left(\mathrm{p}_{\mathrm{Kit}}\left(\mathrm{dK}_{\mathrm{it}} / \mathrm{dt}\right)+\sum_{\mathrm{j}}\left(\mathrm{p}_{\mathrm{Njt}}\left(\mathrm{dN} \mathrm{N}_{\mathrm{jt}} / \mathrm{dt}\right)+\underset{\mathrm{m}}{\sum}\left(\mathrm{p}_{\mathrm{Hmt}} \mathrm{dH} / \mathrm{dt}\right)\right.\right.$

En este caso, la inversión genuina será equivalente a la sumatoria de las variaciones de los diversos tipos de capital valorados por su precio cuenta. Para un periodo t, $\mathrm{K}_{\text {it }}$ es la cantidad del i-ésimo capital artificial, $\mathrm{N}_{\mathrm{jt}}$ la cantidad de la j-ésima forma de capital natural $\mathrm{y} \mathrm{H}_{\mathrm{mt}}$ el m-ésimo tipo de capital humano, siendo sus respectivos precios sombra $\mathrm{p}_{\mathrm{Ki}}, \mathrm{p}_{\mathrm{Nj}}, \mathrm{y} \mathrm{p}_{\mathrm{Hm}}$.

Operacionalmente, la inversión genuina puede evaluarse también mediante la evolución de la riqueza en términos constantes (9), aun cuando sea preferible hacerlo en términos per-cápita.

$\mathrm{W}=\sum_{\mathrm{i}} \mathrm{p}_{\mathrm{Kit}} \mathrm{d}_{\mathrm{Kit}}+\sum_{\mathrm{j}} \mathrm{p}_{\mathrm{Njt}} \mathrm{d}_{\mathrm{Njt}}+\sum_{\mathrm{m}} \mathrm{p}_{\mathrm{Hmt}} \mathrm{d}_{\mathrm{Hmt}}$

Cuando la tasa de crecimiento de la población es relevante y exógena entonces debería calcularse el valor del cambio del capital per-cápita (10), el cual depende de la ratio capital/población (L) y de las tasas de crecimiento del capital y de la población.

$\mathrm{I}_{\mathrm{t}}=\mathrm{p} \Delta(\mathrm{K} / \mathrm{L})=\mathrm{p}(\mathrm{K} / \mathrm{L})[\Delta \mathrm{K} / \mathrm{K}-\Delta \mathrm{L} / \mathrm{L}]$

Aunque la degradación ambiental no es considerada explícitamente en el modelo, parecería que lo es implícitamente. Es de esperar que esta degradación incida en los activos de la economía y, por ende, debería ser capturada en el precio cuenta.

Dado que la inversión genuina utiliza información ex-post, simplemente evalúa si una economía ha estado en la senda hacia el desarrollo sostenible. En otras palabras, el indicador no garantiza que la economía 
en análisis haya presentado un desarrollo sostenible, simplemente indica si ésta ha estado acumulando (o reduciendo) su base productiva para enfrentar un futuro desarrollo que pueda ser sostenible en un futuro.

Por otro lado, la inversión genuina puede no ser sólida en el sentido que la acumulación de capitales haya sido lograda no por el uso eficiente de los recursos sino, por ejemplo, a costa de la deforestación (para ampliar la frontera agrícola y lograr más producción) o revalidación de sus reservas metálicas. Bajo estas condiciones, la economía solo estaría fugazmente en la senda del desarrollo sostenible. Por ello, en términos operativos, la evolución creciente de la riqueza percápita no es condición suficiente para garantizar si la economía en análisis estuvo o no en la senda del desarrollo sostenible. También debe evaluarse paralelamente si esta economía presentó o no fronteras de expansión.

Entonces, una debilidad de la inversión genuina es no asegurar si la economía se encontrará en la senda del desarrollo sostenible en un futuro -incluso- muy cercano. Incluso, no es condición que la economía haya logrado mejoras en sus indicadores sociales. El criterio per se simplemente señala si la economía ha estado acumulando la suficiente riqueza para satisfacer las futuras necesidades que requerirá el desarrollo.

Al margen de todo, quizá la fortaleza más significativa de la inversión genuina es proveer mucha información para analista/ tomadores de decisión sobre los cambios en los activos identificados, por lo tanto, proveer una guía para futuras inversiones (UNU-IHDP y UNEP, 2012).

\section{Materiales y métodos}

A efectos de calcular la Inversión Genuina para Junín durante el periodo 2005-2011 se utilizarán las principales formas de capital identificados: i) artificial $\left(\mathrm{K}^{\mathrm{F}}\right)$, ii) humano $\left(\mathrm{K}^{\mathrm{H}}\right)$, iii) natural $\left(\mathrm{K}^{\mathrm{N}}\right)$, el cual comprende el capital minero $\left(\mathrm{K}^{\mathrm{M}}\right)$, agrícola $\left(\mathrm{K}^{\mathrm{A}}\right)$ y pecuario $\left(\mathrm{K}^{\mathrm{P}}\right)$. Formalmente, la riqueza $(\mathrm{W})$ para un periodo t será:

$\mathrm{W}_{\mathrm{t}}=\mathrm{p}_{\mathrm{t}}^{\mathrm{F}} \mathrm{K}_{\mathrm{t}}^{\mathrm{F}}+\mathrm{p}_{\mathrm{t}}^{\mathrm{N}} \mathrm{K}_{\mathrm{t}}^{\mathrm{N}}+\mathrm{p}_{\mathrm{t}}^{\mathrm{H}} \mathrm{K}_{\mathrm{t}}^{\mathrm{H}}$
Mientras que la Inversión Genuina (I) para ese periodo estará definida por:

$\mathrm{I}_{\mathrm{t}}=\mathrm{p}_{\mathrm{t}}^{\mathrm{F}} \Delta \mathrm{K}_{\mathrm{t}}^{\mathrm{F}}+\mathrm{p}_{\mathrm{t}}^{\mathrm{N}} \Delta \mathrm{K}_{\mathrm{t}}^{\mathrm{N}}+\mathrm{p}_{\mathrm{t}}^{\mathrm{H}} \Delta \mathrm{K}_{\mathrm{t}}^{\mathrm{H}}+\mathrm{v}_{\mathrm{t}}$

donde:

$\mathrm{p}^{\mathrm{F}}$ : Precio cuenta del capital artificial

$\mathrm{p}^{\mathrm{N}}$ : Precio cuenta del capital natural

$\mathrm{p}^{\mathrm{H}}$ : Precio cuenta del capital humano

$\mathrm{v}$ : Drift term (residual)

A continuación, se detalla la forma de estimación de cada uno de ellos.

\section{Capital Artificial}

El primer término del lado derecho (11), equivale al stock de capital (artificial) del país o activo fijo neto. Para obtener la información de $\mathrm{K}^{\mathrm{F}}$, se construyó una serie utilizando el método de inventarios perpetuos:

$\mathrm{K}_{\mathrm{t}}^{\mathrm{F}}=(1-\mathrm{d}) \mathrm{K}_{\mathrm{t}-1}+\mathrm{I}_{\mathrm{t}-1}$

d es la tasa de depreciación asumida en $3 \%$, consistente con la tasa de depreciación anual estimada por Seminario y Beltrán (1998) la cual fluctúa entre 2,5 y 3\%. El termino I es la formación bruta de capital fijo (FBKF). Para calcular el stock de capital inicial $\left(\mathrm{K}_{0}\right)$, se asume que la economía se encuentra en estado estacionario.

Siguiendo la metodología de Easterly y Levine (2001), donde $g$ es la tasa de crecimiento del PIB de Junín. Dado que no existe información estadística de la FBKF de Junín, esta se pudo inferir a partir de dos series existentes: la FBKF del Perú durante el periodo 1994-2012 y los activos fijos de Junín, los cuales fueron obtenidos del BCRP (2013a) e INEI (2013a), respectivamente. La idea fue obtener una serie que al menos siga la misma tendencia del $\mathrm{K}$ del Perú generada por Seminario (2009).

$\mathrm{K}_{0}=\mathrm{I}_{1} /(\mathrm{g}+\mathrm{d})$

Construyendo la FBKF de Junín a partir de extrapolación lineal y usando dicha 
serie en (13) y (14) se estimó el valor de $\mathrm{K}^{\mathrm{F}}$ para Junín. Posteriormente, esta serie fue comparada con el valor de $\mathrm{K}$ del Perú que estimó Seminario (2009), llegando a la conclusión que la serie construida sigue una tendencia similar y por ende es justificable para su respectivo uso.

\section{Capital Natural}

La capital natural esta a su vez conformado por numerosos sub-tipos de capital, siendo los más conocidos minería, bosques, pesquerías, suelo agrícola, suelo pecuario, entre otros. Creciente literatura (UNU-IHDP y UNEP, 2012) sugiere la inclusión de otras formas de capital natural en el cálculo de la riqueza, como, por ejemplo, ecosistemas y recursos hídricos, no obstante dificultad en la medición de ambas constituye una gran limitación para tal inclusión. El caso de Junín no es la excepción.

Para priorizar los tipos de capital natural que serán incluidos en este estudio se tendrán en cuenta la información disponible y la contribución al capital natural al PIB departamental. Según este criterio, minería y suelo agropecuario serán considerados como los capitales naturales comerciales más relevantes, al menos durante el periodo en estudio. No se incluye el sector forestal, puesto que hay información que permita calcular precios cuenta ni stocks. Además, su contribución al PIB departamental es mínima. En las cuentas nacionales, el valor agregado de la actividad forestal está incluida en el valor agregado agropecuario puesto que la contribución de la primera es mínima, la cual -según algunas estimacionesequivale a menos del $1 \%$ del PIB total. La escaza participación del sector forestal en el PIB total tiene que ver en cierta forma con la extracción ilegal cuyo producto no logra ser registrado en las cuentas nacionales.

El capital natural comprende los siguientes capitales: suelo agropecuario $\left(\mathrm{K}^{\mathrm{A}}\right)$ y minería $\left(\mathrm{K}^{\mathrm{M}}\right)$ siendo los respectivos precios cuenta: $\mathrm{P}^{\mathrm{A}}$, y $\mathrm{P}^{\mathrm{M}}$, respectivamente. Para un periodo t, el capital natural total será (15):

$\mathrm{K}_{\mathrm{t}}^{\mathrm{N}}=\mathrm{K}_{\mathrm{t}}^{\mathrm{A}}+\mathrm{K}_{\mathrm{t}}^{\mathrm{M}}$

A continuación, se detalla la forma de estimación tanto de las cuentas físicas como monetarias según el tipo de capital natural.

\section{Suelo agropecuario}

El sector agropecuario de Junín comprende básicamente la actividad agrícola y pecuaria, las mismas que representan el grueso del valor bruto de la producción sectorial. El aporte de la silvicultura es mínimo y por ende, no será analizado en este estudio. Por ello, se asume que el capital suelo agropecuario $\left(\mathrm{K}^{\mathrm{A}}\right)$ en Junín comprende básicamente el capital suelo agrícola $\left(\mathrm{K}^{\mathrm{AG}}\right)$ y capital suelo pecuario $\left(\mathrm{K}^{\mathrm{AP}}\right)$. Formalmente, para un periodo t:

$\mathrm{K}_{\mathrm{t}}^{\mathrm{A}}=\mathrm{K}_{\mathrm{t}}^{\mathrm{AG}}+\mathrm{K}_{\mathrm{t}}^{\mathrm{AP}}$

Puesto que en el Perú se han realizado censos agropecuarios en los años 1994 y 2012, solo existe información física agropecuaria para ambos años.

Dado que el precio cuenta del sector agropecuario no está disponible a nivel nacional ni departamental, tuvo que ser estimado. El sector agrícola de Junín comprende numerosos cultivos. Si bien los precios de mercado de todos los productos agrícolas están disponibles, los costos de producción no lo están. Por ello se decidió utilizar el excedente de explotación agropecuario $\left(\mathrm{EE}^{\mathrm{A}}\right)$ como aproximación del beneficio bruto agropecuario departamental.

Para descomponer este excedente en el excedente de explotación agrícola $\left(\mathrm{EE}^{\mathrm{AG}}\right)$ y excedente de explotación pecuario $\left(\mathrm{EE}^{\mathrm{AP}}\right)$ se requiere los factores de contribución del sector agrícola y pecuario $(\alpha$ y $\beta$, respectivamente) al excedente de explotación agropecuario total departamental. Para un periodo $t$ (17):

$\mathrm{EE}_{\mathrm{t}}^{\mathrm{A}}=\alpha\left(\mathrm{EE}_{\mathrm{t}}^{\mathrm{AG}}\right)+\beta\left(\mathrm{EE}_{\mathrm{t}}^{\mathrm{AP}}\right)$

Claramente, $\alpha+\beta=1$. La estimación de todas estas variables es detallada en Orihuela (2014). Una vez estimados los excedentes de explotación agrícola y pecuaria, se procede a calcular los correspondientes precios cuenta para el horizonte en estudio (18) y (19): 


$$
\begin{aligned}
& \mathrm{P}_{\mathrm{t}}^{\mathrm{AG}}=\alpha\left(\mathrm{EE}_{\mathrm{t}}^{\mathrm{A}}\right) /\left(\mathrm{S}_{\mathrm{t}}^{\mathrm{AG}}\right) \\
& \mathrm{P}_{\mathrm{t}}^{\mathrm{AP}}=\beta\left(\mathrm{EE}_{\mathrm{t}}^{\mathrm{A}}\right) /\left(\mathrm{S}_{\mathrm{t}}^{\mathrm{AP}}\right)
\end{aligned}
$$

Nótese que los precios cuentan - dada la forma de estimación propuesta - equivalen a beneficios promedio y no beneficios marginales.

No hay estudios a nivel local que hayan evaluado los retornos de escala del sector agrícola y/o pecuario. Conforme a INEI y MINAG (2013) la mayor parte de las unidades de producción agropecuarias en el Perú son relativamente pequeñas (menores a 5 ha) cuyo número ha sido creciente durante el periodo de estudio, lo cual sugiere un fraccionamiento cada vez mayor de estas unidades. Esto ha ocurrido en la sierra puesto que en la costa ocurrió un fenómeno inverso: concentración de tierras (Remy y de los Ríos, 2012). En este contexto, parece razonable asumir la presencia de retornos de escala constantes en el sector agropecuario de Junín (sierra).

Así, estimar el precio cuenta como un beneficio promedio (en lugar de beneficio marginal) no debería generar distorsiones significativas en los resultados. Una vez obtenidos los dos precios cuenta, la riqueza del suelo agropecuario $\left(\mathrm{W}^{\mathrm{A}}\right)$ será equivalente a la sumatoria del capital suelo agrícola y suelo pecuario valorados por sus respectivos precios cuenta (en este caso, $\mathrm{P}^{\mathrm{AG}}$ y $\mathrm{P}^{\mathrm{AP}}$, respectivamente).

$\mathrm{W}_{t}^{\mathrm{A}}=\mathrm{P}_{t}^{\mathrm{AG}} \mathrm{K}_{t}^{\mathrm{AG}}+\mathrm{P}_{t}^{\mathrm{AP}} \mathrm{K}_{t}^{\mathrm{AP}}$

Todas las cifras monetarias fueron convertidas a soles constantes 1994 utilizando el deflactor implícito del PIB agropecuario.

\section{Capital Minero}

El sector minero de Junín es polimetálico de manera que comprende la extracción, y concentración (según el metal) de oro, plata, cobre, plomo, y zinc, siendo éste último, el que más contribuye al valor bruto de la producción sectorial. De esta forma, el capital minero $\left(\mathrm{K}^{\mathrm{M}}\right)$ está conformado por capitales vinculados al oro, plata, cobre, zinc, y plomo, denotados respectivamente por: $\mathrm{K}^{\mathrm{MO}}, \mathrm{K}^{\mathrm{MP}}$,
$\mathrm{K}^{\mathrm{MC}}, \mathrm{K}^{\mathrm{MZ}}, \mathrm{y} \mathrm{K}^{\mathrm{ML}}$, respectivamente. Para un periodo $t(21)$ :

$\mathrm{K}_{\mathrm{t}}^{\mathrm{M}}=\mathrm{K}_{\mathrm{t}}^{\mathrm{MO}}+\mathrm{K}_{\mathrm{t}}^{\mathrm{MP}}+\mathrm{K}_{\mathrm{t}}^{\mathrm{MC}}+\mathrm{K}_{\mathrm{t}}^{\mathrm{MZ}}+\mathrm{K}_{\mathrm{t}}^{\mathrm{ML}}$

Según Ministerio de Energía y Minas (2013a), los metales que poseen reservas probadas y probables en Junín son: oro, plata, cobre, plomo, zinc y hierro. Las estadísticas de estas reservas por metal para todo el periodo de estudio fueron obtenidas de esa fuente. Cabe mencionar que Junín posee reservas probadas y probables de hierro, las cuales si bien forman parte del capital natural no son valoradas puesto que estas reservas no contribuyeron al bienestar humano local durante el periodo de estudio, de manera que su precio cuenta fue cero. El precio cuenta de cada metal equivale al beneficio marginal social de extraer (producir) fue estimado por Orihuela (2014).

Todas las cifras monetarias fueron convertidas a soles constantes 1994 usando el deflactor implícito del PIB Minero. El valor del capital minero (riqueza) será equivalente a la sumatoria de los capitales que la conforman multiplicados por sus respectivos precios cuenta.

$$
\begin{aligned}
& \mathrm{W}_{t}^{\mathrm{M}}=\mathrm{P}_{\mathrm{t}}^{\mathrm{MO}} \mathrm{K}_{\mathrm{t}}^{\mathrm{MO}}+\mathrm{P}_{\mathrm{t}}^{\mathrm{MP}} \mathrm{K}_{\mathrm{t}}^{\mathrm{MP}}+\mathrm{P}_{\mathrm{t}}^{\mathrm{MC}} \mathrm{K}_{\mathrm{t}}{ }^{\mathrm{MC}}+\mathrm{P}_{\mathrm{t}}^{\mathrm{MZ}} \mathrm{K}_{\mathrm{t}}^{\mathrm{MZ}} \\
& +\mathrm{P}_{\mathrm{t}}^{\mathrm{MH}} \mathrm{K}_{\mathrm{t}}^{\mathrm{MH}}+\mathrm{P}_{\mathrm{t}}^{\mathrm{ME}} \mathrm{K}_{\mathrm{t}}^{\mathrm{ME}}+\mathrm{P}_{t}^{\mathrm{ML}} \mathrm{K}_{\mathrm{t}}^{\mathrm{ML}}
\end{aligned}
$$

\section{Capital humano}

A priori, no es sencillo imputar un valor a la vida humana, lo cual puede resultar incluso anti-ético. Sin embargo, el objetivo del valorar la vida humana es medir monetariamente como las destrezas y habilidades de una sociedad pueden ser útiles para su economía. Eso es lo que generará beneficios futuros. Esta es una medida de opulencia de una economía. En la literatura existen al menos dos métodos para valorar el capital humano: valor de una vida estadística (VVE) y método del capital humano $(\mathrm{MCH})$.

El primero (VVE) consiste en medir la disposición a pagar por evitar una muerte. El VVE es calculado dividiendo la disposición de pago marginal para reducir el riesgo de muerte por el tamaño de la reducción de riesgo. Este método ofrece el valor más 
alto de un daño a la salud. El segundo método $(\mathrm{MCH})$ estima el costo indirecto de productividad perdida mediante el valor de los ingresos futuros que serían perdidos.

Desde una perspectiva microeconómica, el VVE constituiría el método correcto puesto que captura las preferencias de la sociedad por reducir el riesgo de muerte, e indirectamente, por la vida humana. En el Perú no hay estudios o estimaciones sobre la disposición de pago por reducir el riesgo en mención. Si bien este dato puedo extrapolarse de otros países (mediante el método de transferencia de beneficios), se incurría en una distorsión al introducir las preferencias de una sociedad en otra.

Quizá por este motivo, aunado a la disponibilidad de información que se requiere y a la sencillez de su aplicación, es que el valor del capital humano ha sido frecuentemente estimado mediante el $\mathrm{MCH}$. Los estudios más conocidos sobre inversión genuina y ahorro genuino, tales como Arrow et al. (2012) y World Bank (2011) utilizaron este método. Así, el método tiene la ventaja de que los resultados sean comparables con trabajos similares. Por estos motivos, el método será aplicado en este estudio.

En un momento t, el capital humano de un individuo h está determinado por (23), donde $\alpha$ es la tasa promedio de retorno de la educación y el promedio de años de estudio logrados de la población mayor a 14 años (aquella en condiciones de trabajar).

$h_{t}=e^{t \theta t}$

El stock agregado de capital humano es denotado por $\mathrm{H}$, equivalente a la multiplicación del capital humano individual por la población total mayor de 15 años (PEA).

$\mathrm{H}_{\mathrm{t}}=\mathrm{h}_{\mathrm{t}}\left(\mathrm{PEA}_{\mathrm{t}}\right)$

Como todo activo, el capital humano también se deprecia cuando algún individuo muere. Es muy difícil medir este efecto. Una gruesa aproximación consiste en ajustar este stock agregado por la tasa bruta de mortalidad (TBM). Entonces, el capital humano ajustado, HA, en un periodo t será:
$\mathrm{HA}_{\mathrm{t}}=\mathrm{H}_{\mathrm{t}}\left(1-\mathrm{TBM}_{\mathrm{t}}\right)$

El valor acumulado de una unidad de capital humano o su precio, $\mathrm{P}_{\mathrm{H}}$, se calcula mediante la sumatoria descontada de los ingresos $\left(\mathrm{R}_{\mathrm{t}}\right)$ que cada unidad genera para el número promedio de años restantes de trabajo, $\mathrm{T}$.

$P H t=\int_{0}^{T} R_{t} e^{-\alpha t} d t$

0

Finalmente, el valor del stock de capital agregado de la economía es representado por (27)

$\mathrm{V}_{\mathrm{t}}=\mathrm{P}_{\mathrm{t}} \mathrm{HA}_{\mathrm{t}}$

Yamada y Castro (2010) reconocen que la tasa de retorno de la educación en el Perú es de $10 \%$ cuando se utiliza la especificación tradicional de la ecuación de Mincer. Ellos identifican que los supuestos de tal especificación no se verifican en el caso peruano. Por ello construyen especificaciones alternativas encontrando diferencias significativas en los resultados (en comparación a la clásica especificación). Sus resultados sugieren que la tasa de retorno fluctúa entre $3,5 \%$ y casi $30 \%$, dependiendo del nivel de instrucción básica o superior, o cursar un nivel educativo o completarlo.

Si bien los resultados de Yamada y Castro (2010) sugieren que la tasa de retorno de la educación oscila ampliamente dependiendo del caso, sería necesario un estudio muy fino para determinar un promedio ponderado para el caso peruano, y sobre todo, para cada año del periodo en análisis. De manera conservadora este estudio propone adoptar tasa de $10 \%(\alpha=0,10)$, la cual va en línea con el promedio nacional obtenido por Armendáriz et al. (2011) y similar a los resultados de Barco y Vargas (2010). Los datos anuales de fueron obtenidos de INEI (2012b).

De INEI (2012c) se obtuvo la información de PEA y TBM. Dado que esta información es quinquenal fue necesario estimar una tasa de crecimiento promedio 
para proyectar la serie requerida. Para calcular anualmente el termino R, se dividió las remuneraciones totales de Junín entre la PEA ocupada mayor de 14 años. No hay estadísticas sobre la evolución de estas remuneraciones, habiéndose publicado (hasta donde se conoce) esta remuneración sólo para los años 1993 y 2007 (INEI, 1994; 2008). Por ende, se estimó una tasa de crecimiento promedio implícita a fin de proyectar tales remuneraciones para todo el periodo en estudio.

El número de años restantes de trabajo, $\mathrm{T}$, se calculó como la diferencia entre la esperanza de vida, EV, y la edad promedio de la población, EP de Junín. Es decir, T= EV-EP. La información de EV fue obtenida de la página web de INEI. Esta fuente ofrece también información poblacional por edades. Sólo se consideró los grupos de edades de 15-19 hasta 60-64 años. Luego se obtuvo el promedio ponderado.

\section{Drif Term}

El último término del lado derecho de la expresión (12), es el drift term, el cual equivale a la contribución en la inversión genuina que no es generada por los capitales considerados. Normalmente se utiliza la Productividad Total de Factores (PTF) como una aproximación al drift term. La PTF equivale al residual de la función de crecimiento de Solow. En el caso de una función tradicional de Solow, este residual contiene aquellos factores de la producción diferentes al capital y trabajo. Por lo tanto, en el contexto de crecimiento del producto, la PTF es entendida como la productividad, mientras que, en el caso de la inversión genuina, sería aquellos capitales que inciden o contribuyen en el cambio de la riqueza total, por ejemplo, el capital institucional. Esta cifra fue obtenida de Orihuela (2014).

\section{Resultados y discusión}

De los resultados (Tabla 1), se desprende que la mayor fuente de riqueza recae largamente en el capital artificial, el cual es la base para sostener todas las actividades económicas, incluyendo comercio, manufactura, transportes, entre otros. El segundo lugar, lo ocupa el capital natural, siendo el capital minero la principal riqueza. La riqueza del capital humano ha ocupado el tercer lugar, superando al capital suelo agropecuario. $\mathrm{Si}$ bien la actividad agropecuaria es una importante fuente de trabajo, de manera que su aporte al PIB departamental ha sido significativo, su contribución a la riqueza departamental ha sido más bien reducida. Esto sería una evidencia de que el aporte del sector agropecuario no sería tan significativo en el bienestar humano futuro de Junín. Corroborar esta hipótesis requiere estudios adicionales .

Tabla 1: Riqueza de Junín (millones de nuevos soles 1994) durante el periodo 1994-2011 por tipo de capital

\begin{tabular}{ccccccc}
\hline & $\begin{array}{c}\text { Suelo } \\
\text { Año }\end{array}$ & $\begin{array}{c}\text { Sgrícolo } \\
\text { Pecuario }\end{array}$ & \multicolumn{3}{c}{ Minero Artificial Humano } & Total \\
\cline { 2 - 7 } & $\mathrm{P}_{\mathrm{A}} \mathrm{K}_{\mathrm{A}}$ & $\mathrm{P}_{\mathrm{P}} \mathrm{K}_{\mathrm{P}}$ & $\mathrm{P}_{\mathrm{M}} \mathrm{K}_{\mathrm{M}}$ & $\mathrm{P}_{\mathrm{F}} \mathrm{K}_{\mathrm{F}}$ & $\mathrm{P}_{\mathrm{H}} \mathrm{K}_{\mathrm{H}}$ & $\mathrm{W}$ \\
\hline 1994 & 567 & 39 & $\mathrm{Nd}$ & 49,501 & 1,832 & 51,940 \\
1995 & 575 & 39 & $\mathrm{Nd}$ & 50,520 & 2,145 & 53,280 \\
1996 & 584 & 39 & $\mathrm{Nd}$ & 52,125 & 2,513 & 55,260 \\
1997 & 593 & 39 & $\mathrm{Nd}$ & 53,552 & 2,945 & 57,129 \\
1998 & 601 & 39 & $\mathrm{Nd}$ & 55,282 & 3,452 & 59,374 \\
1999 & 611 & 38 & $\mathrm{Nd}$ & 57,011 & 4,049 & 61,708 \\
2000 & 620 & 38 & $\mathrm{Nd}$ & 58,546 & 4,751 & 63,955 \\
2001 & 629 & 38 & $\mathrm{Nd}$ & 59,925 & 4,834 & 65,426 \\
2002 & 638 & 38 & $\mathrm{Nd}$ & 60,997 & 5,051 & 66,725 \\
2003 & 648 & 38 & $\mathrm{Nd}$ & 62,110 & 5,353 & 68,149 \\
2004 & 658 & 38 & $\mathrm{Nd}$ & 63,282 & 5,758 & 69,735 \\
2005 & 668 & 37 & 3,640 & 64,571 & 5,525 & 74,441 \\
2006 & 678 & 37 & 4,110 & 66,007 & 5,969 & 76,801 \\
2007 & 688 & 37 & 8,737 & 67,656 & 6,033 & 83,150 \\
2008 & 698 & 37 & 5,488 & 69,787 & 6,245 & 82,256 \\
2009 & 709 & 37 & 6,946 & 73,292 & 6,402 & 87,385 \\
2010 & 719 & 37 & 7,459 & 76,028 & 6,427 & 90,670 \\
2011 & 730 & 36 & 7,555 & 79,587 & 6,672 & 94,580 \\
\hline
\end{tabular}

Nd: no disponible 
La riqueza total aumentó durante el periodo en análisis (27\%), es decir el departamento Junín ha estado acumulando capital (en términos monetarios). Otra forma de interpretar los resultados es estimando la inversión genuina mediante la expresión (10). Un mejor análisis de la riqueza debe incorporar el crecimiento de la población.

Conforme al Tabla 2, la riqueza per-cápita también creció durante el periodo 20052011 (18\%), no obstante el crecimiento del PIB per-cápita fue superior (33\%). En teoría, si la tasa de crecimiento del a riqueza del PIB con iguales implicaría que la economía local estaría cercana a un nivel óptimo En un modelo sencillo con una senda de crecimiento óptima, para que el crecimiento del stock de capital sea el mismo que el crecimiento del PIB se requiere que la función de producción debe presentar retornos de escala constantes (UNU-IHDP y UNEP, 2012). En teoría, si una economía altamente dependiente de los recursos naturales no ha hecho un manejo apropiado de tales recursos (por ejemplo, reinversión de rentas en otras formas de capital productivo), es de esperar niveles crecientes del PIB a costa de una merma de la base productiva, constituida principalmente por capital natural. Claramente, este no es el caso de Junín conforme a las tasas obtenidas.
Incorporando la población e incluso la PTF (cambio tecnológico, capital institucional, etc.), los resultados siguen siendo positivos. Esto sugiere que este departamento mantiene intactas las capacidades para poder generar bienestar humano en el futuro. En otras palabras, esta economía departamental ha estado en la senda del desarrollo sostenible.

Las conclusiones de la Tabla 1 son contrarias a los obtenidas por World Bank (2006; 2011) y Hamilton y Atkinson (2006) en donde la riqueza en diversos bloques de países (años 2000 y 2005) se concentró en el capital intangible (equivalente al capital humano y capital institucional), constándose que el desarrollo económico estuvo basado en una rápida acumulación (monetaria) del capital artificial e intangible a costa de un excesivo agotamiento del capital natural.

Esto no fue el caso de Junín, en donde hubo acumulación en la riqueza de todos los capitales que conforman la base productiva departamental, excepto el capital natural pecuario. Más aún, la contribución del capital artificial con respecto al capital total, se redujo levemente mientras que el aporte del capital natural aumentó (Tabla 3). En términos per-cápita, esta acumulación ha sido la menor en comparación al resto de capitales (Tabla 4).

Tabla 2: Tasas de crecimiento (\%) de la riqueza per-cápita, ajustado por cambio tecnológico

(PTF) durante el periodo 2005-2011

\begin{tabular}{cccccc}
\hline $\begin{array}{c}\text { Tasa de } \\
\text { crecimiento decrecimiento de } \\
\text { la riqueza } \\
\text { la población }\end{array}$ & $\begin{array}{c}\text { Tasa de } \\
\text { crecimiento de } \\
\text { laqueza per- } \\
\text { cápita }\end{array}$ & $\begin{array}{c}\text { Tasa de } \\
\text { crecimiento } \\
\text { del PTF }\end{array}$ & $\begin{array}{c}\text { Tasa de crecimiento } \\
\text { de la riqueza } \\
\text { per-cápita, } \\
\text { incorporando PFT }\end{array}$ & $\begin{array}{c}\text { Tasa de } \\
\text { crecimiento } \\
\text { del PIB per- } \\
\text { cápita }\end{array}$ \\
\hline$(1)$ & $(2)$ & $(3)=(1)-(2)$ & $(4)$ & $(5)=(3)+(4)$ & $(6)$ \\
27,1 & 7,5 & 19,6 & 5,0 & 24,6 & 33,3 \\
\hline
\end{tabular}

Tabla 3: Composición de la riqueza total (\%) por tipo de capital en los años 2005 y 2011

\begin{tabular}{ccccccc}
\hline Años & $\mathrm{P}_{\mathrm{A}} \mathrm{K}_{\mathrm{A}}$ & $\mathrm{P}_{\mathrm{P}} \mathrm{K}_{\mathrm{P}}$ & $\mathrm{P}_{\mathrm{M}} \mathrm{K}_{\mathrm{M}}$ & $\mathrm{P}_{\mathrm{F}} \mathrm{K}_{\mathrm{F}}$ & $\mathrm{P}_{\mathrm{H}} \mathrm{K}_{\mathrm{H}}$ & $\mathrm{W}$ \\
\hline 2005 & 0,9 & 0,1 & 4,9 & 86,7 & 7,4 & 100 \\
2011 & 0,8 & 0,0 & 8,0 & 84,1 & 7,1 & 100 \\
\hline
\end{tabular}

Tabla 4: Medidas de riqueza per-cápita (soles 1994) según capital durante el periodo 1994-2011

\begin{tabular}{cccccc}
\hline Año & $\mathrm{P}_{\mathrm{A}} \mathrm{K}_{\mathrm{A}} / \mathrm{P}$ & $\mathrm{P}_{\mathrm{P}} \mathrm{K}_{\mathrm{P}} / \mathrm{P}$ & $\mathrm{P}_{\mathrm{M}} \mathrm{K}_{\mathrm{M}} / \mathrm{P}$ & $\mathrm{P}_{\mathrm{F}} \mathrm{K}_{\mathrm{F}} / \mathrm{P}$ & $\mathrm{P}_{\mathrm{H}} \mathrm{K}_{\mathrm{H}} / \mathrm{P}$ \\
\hline 2005 & 558 & 31 & 3,042 & 53,971 & 4,618 \\
2011 & 568 & 28 & 5,876 & 61,898 & 5,189 \\
\hline $\mathrm{T} / \mathrm{C}$ & 2 & -9 & 93 & 15 & 12 \\
\hline
\end{tabular}


Aun cuando estos resultados corroboren que Junín ha estado en la senda del desarrollo sostenible, conforme a la metodología utilizada, esto no garantiza que los altos niveles de crecimiento del PIB per-cápita registrados durante el periodo 2005-2011 se mantengan en el futuro cercano. Por un lado, altas tasas de crecimiento del ingreso requieren al mismo tiempo niveles de acumulación significativa de la riqueza. Esto no ha ocurrido en Junín. Además, si bien la riqueza artificial fue la más significativa en la riqueza total departamental, su aumento $(15 \%)$ ha sido el menor en comparación a otros capitales.

\section{¿Realmente el departamento Junín está en condiciones de mantener su desarrollo?}

Como fue señalado, la acumulación de riqueza per se no es una condición suficiente para lograr un desarrollo futuro. Lo ideal es que esta acumulación sea lograda a partir de una adecuada gestión de los recursos (base productiva) que dispone la economía, es decir, que sea resultado de la eficiencia. Asimismo, se requiere un capital institucional que permita realmente articular de la mejor forma esta base productiva.

La estimación de la PTF departamental, como una aproximación del aporte del capital institucional en el periodo 20052011, ha sido poco significativa (5\%), lo cual sugiere que este capital -y otros no considerados- han contribuido levemente en la economía departamental. Si bien es cierto que esta PTF también comprende otros factores que inciden en el crecimiento económico departamental (por ejemplo, productividad o informalidad), es de esperar que el capital institucional haya aportado alguna contribución. Un análisis muy fino podría medir el grado de contribución.

El capital intangible está muy vinculado al capital artificial, el cual es una especie de capital articulador, ya que cumple la función de soporte para la dinámica económica departamental. El capital artificial incluso facilita la creación y/o crecimiento de otros capitales. No se dispone de estudios que permitan evaluar si este leve crecimiento ha sido el suficiente para sostener o articular adecuadamente el resto de capitales. Probablemente, la abultada y creciente brecha de inversión nacional en servicios públicos (Perroti y Sanchez,
2011) justifique su escaso crecimiento y por ende, requiera de una mayor inversión. Armendáriz et al. (2011) sostienen que la calidad de la infraestructura vial (asfaltado) constituye una de las debilidades de Junín hacia el desarrollo. Esto está vinculado a deficiencias en la gestión para ejecutar el gasto público. Según estimaciones de los autores, la informalidad en Junín es alta e incluso excede al promedio nacional $(75,6 \%$ de la PEA).

Sobre el capital humano es poco lo que se puede desprender. Si bien la metodología de su cálculo permite inferir el valor del capital aumentó en el periodo de estudio no permite inferir mucho a determinar si hubo una mejora en la calidad. Armendáriz et al. (2011) encontró que los retornos a la educación de Junín (para el año 2007) fueron similares al promedio nacional, concluyendo que la educación -en calidad y cantidad- no ha limitado el crecimiento económico del departamento en mención. Los hallazgos de estos autores sugieren que el capital humano de Junín no ha sido una limitante al desarrollo, aunque en el futuro cercano puede llegar a serlo.

Aun cuando el PIB per-cápita departamental sea creciente, no queda claro si el (creciente) capital humano que ha presentado Junín durante el periodo analizado constituye una limitación o ventaja para el crecimiento económico. Si este (creciente) capital ha concentrado gran parte de la riqueza departamental, es de esperar que su aporte haya sido muy significativo y que sus niveles actuales sean en cierta forma cercanos a los óptimos. Sería necesario un análisis profundo para determinar la brecha (si la hubiera) de capital humano en este departamento.

De otro lado, aunque la riqueza minera ha aumentado considerablemente durante el periodo de estudio (48\%), las reservas del año 2011 alcanzarían apenas para los próximos 20 años, asumiendo una tasa de extracción anual similar a la registrada el año 2011 (Tabla 5). Si bien las reservas mineras se comportan como un flujo de recursos que se incrementa en la medida que se invierte en exploración y desarrollo, existe un límite o tamaño físico desconocido que será limitante en el futuro -al menos para Junínde no reinvertirse las rentas de los recursos mineros en otras formas de capital. 
Tabla 5: Reservas totales por metal en el año 2011

\begin{tabular}{ccccc}
\hline Metal & Reservas totales* & Unidad & $\begin{array}{c}\text { Producción } \\
\text { del año 2011 }\end{array}$ & $\begin{array}{c}\text { Años } \\
\text { remanentes }\end{array}$ \\
\hline Zinc & 2848,413 & TMF & 199,446 & 14 \\
Plomo & 594,033 & TMF & 35,079 & 17 \\
Cobre & 190,190 & TMF & 8,853 & 21 \\
Oro & 123,790 & Oz & 33,647 & 4 \\
Plata & 313295,440 & Oz & 14318,000 & 22 \\
\hline
\end{tabular}

*Equivalente a reservas probadas más reservas probables Fuente: Ministerio de Energía y Minas (2013a; 2013b)

No hay estadísticas sobre el nivel de degradación y/o erosión del suelo agropecuario en Junín, de manera que pueda evaluarse las limitaciones de este capital hacia el futuro. En conclusión, las perspectivas endógenas favorables de la base productiva de Junín permitirían mantener niveles de ingreso similares a los presentados en el periodo analizado. En otras palabras, la acumulación de base productiva, permitiría mantener un nivel de bienestar humano no decreciente para las futuras generaciones.

\section{Fronteras de expansión}

La economía departamental posee un intangible significativo y ha presentado una acumulación significativa de riqueza en todos sus capitales identificados, especialmente en capital artificial. Sin embargo, no queda claro si el crecimiento económico (ya sea en términos de riqueza o ingreso) logrado Junín ha sido a costa -o en parte- de una expansión de fronteras. Para ello deberían evaluarse -entre otras- las condiciones de Barbier (2007).

Para este caso particular, las condiciones deberían recaer en i) evaluación de la forma de aumento de cada uno de los capitales que conforman la base productiva departamental (eficiencia), con particular énfasis en el capital natural, y ii) manejo sostenible de los recursos naturales. El término "manejo sostenible" no garantiza necesariamente maximizar el bienestar humano actual o futuro. En el caso de los recursos no renovables, el manejo sostenible debe consistir no sólo en obtener una renta óptima por su uso, sino también reinvertirlas en los sectores más dinámicos y productivos (Barbier, 2007). Nótese que desde un punto de vista económico sólo es importante que el bienestar humano -provisto por el recurso- sea no decreciente, puesto que el recurso no renovable inevitablemente tenderá al agotamiento. En el caso de los recursos renovables, es común interpretar el manejo sostenible como aquel que genere ingresos no decrecientes sin amenazar la tasa de regeneración natural del recurso. Esto no necesariamente brindaría el mayor bienestar humano posible en el presente o futuro, lo cual dependerá -entre otras cosas- del costo de oportunidad. Asumiendo que no existe un mejor uso del suelo agropecuario (para otras actividades), entonces el manejo sostenible debería garantizar que el potencial del suelo agropecuario sea no decreciente en el tiempo.

\section{Expansión del suelo agropecuario}

No hay estadísticas oficiales sobre la evolución anual de la superficie cultivada en Junín. Esta tuvo que ser inferida a partir de la tasa de crecimiento anual implícita de los registros de los años 1994 y 2012, en los cuales se realizaron los censos agrarios INEI (1994; 2013c). Los resultados sugieren que hubo una leve expansión de esta superficie ( $7 \%$ durante el periodo, $1 \%$ anual), la cual estuvo acompañada de productividad agrícola creciente, medida como el ratio ingreso/superficie cosechada $\left(\mathrm{VBP}^{\mathrm{AG}} / \mathrm{S}^{\mathrm{AG}}\right)$ en términos constantes 1994. Este crecimiento acumulado de la frontera ha sido similar al crecimiento poblacional de Junín durante el mismo periodo (7\%), Esta tasa fue calculada a partir de la población estimada en los años 1993 y 2008. Se obtuvo una tasa implícita de crecimiento para este periodo, la cual fue posteriormente utilizada para proyectar la población en el periodo de estudio, pero largamente inferior al crecimiento acumulado del VBP agrícola (43\%). 


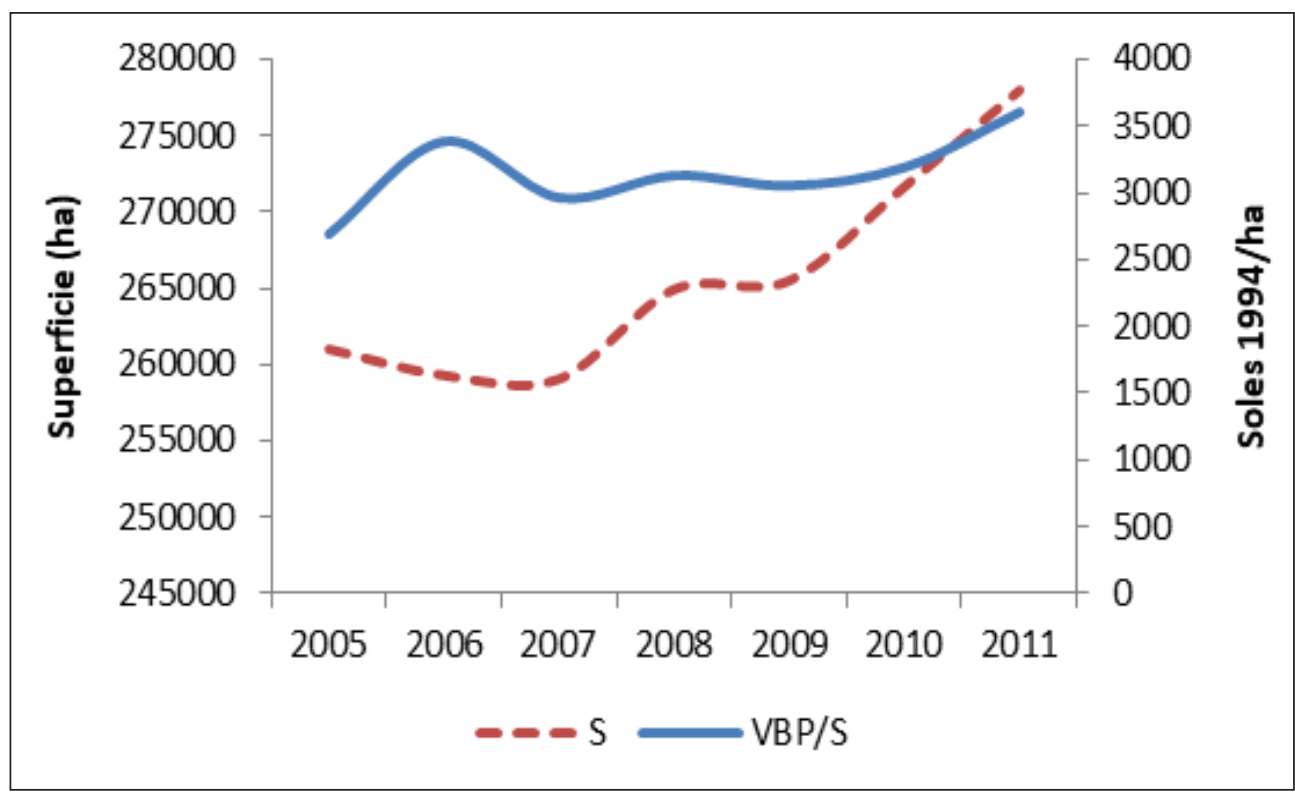

Figura 1: Productividad agrícola y superficie cosechada de Junín

Fuente: INEI (1994; 2013b)

En conclusión, hubo una leve expansión de frontera agrícola, no obstante, la productividad del sector agrícola aumentó más que proporcionalmente (Figura 1).

En base a los resultados de este estudio, los metales y el suelo agropecuarios constituyen los principales recursos naturales de Junín. Un adecuado manejo de estos recursos debería lograr el máximo valor presente de los futuros beneficios que tales recursos podrían generar. Naturalmente, este objetivo responde a un criterio estrictamente económico y es consistente con el enfoque de sostenibilidad débil.

\section{Suelo Agropecuario}

No hay la certeza que los beneficios agropecuarios hayan sido los máximos posibles. En general, la variabilidad de estos beneficios es muy alta, puesto que depende no sólo del nivel de tecnología y capital utilizado en la producción, sino además de las expectativas de precios y clima, las cuales son muchas poseen un carácter volátil. Sin embargo, los niveles crecientes de la productividad agrícola presentados en Junín durante el periodo de estudio pueden ser considerados como una evidencia un manejo adecuado, pero no necesariamente óptimo de este capital.

No hay estadísticas de los niveles de degradación ni erosión del suelo agropecuario en Junín. Tampoco se dispone de información que permita inferir si estos efectos han sido o están siendo mitigados, de manera que pueda concluirse si tales amenazas son realmente significativas que manera que constituyan limitantes al desarrollo local. Presumiblemente, el aumento en la productividad agrícola (Figura 1) compensó tales amenazas.

\section{Recursos no renovables: metales}

En el caso de los recursos no renovables, la estrategia -según la economía de recursos naturales- consiste en determinar un nivel de extracción óptimo y/o obtener una regalía óptima. El dueño de los recursos naturales tiene el derecho de imponer una regalía por su uso. Este es el caso de la minería peruana. En el caso del suelo agropecuario no se requiere una regalía dado que el propietario es un agente privado. Esta regalía debería ser reinvertida en otros tipos de capital, no solo para diversificar sino para conseguir que el nivel de bienestar generado por el recurso no 
renovable sea no decreciente en el tiempo, aun cuando este recurso haya sido agotado.

Las rentas de los recursos naturales no solo deben ser óptimas (es decir, aquellas que maximizan el flujo descontado de ingresos que percibiría la Nación), sino también, reinvertidas apropiadamente en otros tipos de capital. Hay evidencia que estas rentas no han sido óptimas en el Perú (Cantuarias y Orihuela, 2010) y probablemente también en Junín. Presumiblemente, aun cuando estas rentas -en parte- son reinvertidas en capital artificial y posiblemente en capital humano, no están siendo reinvertidas adecuadamente, no obstante este análisis escapa al objetivo de presente estudio. Boza (2006) encuentra que las rentas de la minería son destinadas a gastos irrelevantes para el desarrollo de las comunidades en donde el metal es encontrado.

Diversos estudios (GTZ, 2008; Boza, 2006) sugieren que este ha sido el común denominador en el gasto de los recursos provenientes de la minería en el Perú. En todo caso, serían necesarios mayores estudios que corroboren esta hipótesis para el caso particular de Junín. Los efectos de un manejo inapropiado de las regalías de los recursos no renovables podrían pasar la cuenta incluso en el futuro cercano -aun cuando el capital minero no sea el principal componente de la base productiva-puesto que la dinámica económica podría estar siendo muy dependiente del efecto multiplicador de la minería. La cuantificación de este impacto escapa al alcance de este, aunque debe ser materia de futuros estudios.

Tampoco queda claro si el agotamiento del capital natural (no renovable) ha estado siendo compensado con la incorporación de otras formas de capital a la base productiva departamental, tal cual sugiere el enfoque de sostenibilidad débil. Esta hipótesis también debería ser contrastada en futuros estudios.

\section{Conclusiones}

En base al criterio de la inversión genuina, los resultados demuestran que Junín se encontró en la senda del desarrollo sostenible durante el periodo 2005-2011. El aumento en casi la totalidad de su base productiva constituye un caso atípico para una economía de un país subdesarrollado, la cual suele acumular capital artificial y humano a costa del agotamiento del capital natural. Esto no ocurrió en Junín.

Si bien Junín presentó una inversión genuina positiva e indicadores crecientes de riqueza per-cápita (excepto en el capital pecuario), ello no garantiza que en el futuro la base productiva siga creciendo endógenamente $\mathrm{y} / \mathrm{o}$ que la evolución del PIB departamental sea creciente en términos reales. Esto dependerá tanto de aspectos institucionales -los cuales asignan los recursos- como de otras condiciones exógenas futuras (por ejemplo, crisis internacionales).

El capital más importante de Junín es -de lejos- el capital artificial que si bien concentra una fracción muy significativa del total, su crecimiento durante el periodo de estudio ha sido sustancialmente menor en comparación al crecimiento de otros capitales. Algunos estudios sobre inversión nacional sugieren aún existe una brecha significativa. El caso de Junín podría ser el mismo, puesto que no se sabe si el nivel actual del capital artificial es el apropiado. Esto debería ser materia de futuros estudios.

Se encontró que el capital natural más relevante no es el agrícola ni pecuario sino el capital minero, cuyo manejo -según las evidencias- parecería no ser el más apropiado, lo cual limitaría las futuras posibilidades de desarrollo de Junín.

Puesto que la tasa de crecimiento del PIB per-cápita excedió la tasa de crecimiento de la riqueza per-cápita, se requiere incrementar capitales en Junín. La prioridad debería recaer sobre el capital artificial y capital humano. Posiblemente, inversiones para mejorar la calidad de las vías terrestres y la construcción de otras vías (por ejemplo, en ferrocarriles) constituirían posibles opciones para el caso del aumento del capital artificial.

Debe fomentarse estudios específicos sobre brechas en infraestructura y capital humano. Esto es el punto de partida para implementar políticas públicas puntuales. Estos estudios deberían considerar no sólo el beneficio para Junín sino para los departamentos vinculados en gran medida con su economía (por ejemplo, Lima).

\section{Agradecimientos}

Este articulo está basado en el estudio 
“¿La región Junín ha estado en la senda del desarrollo sostenible? Evidencia del periodo 2005-2011", el cual fue financiado por el Fondo de Estudios y Consultorías Belga-Peruano (FECBP) y operado por el Consorcio de Investigación Económica y Social (CIES). Los autores agradecen a estas instituciones. Cualquier error u omisión es responsabilidad exclusiva de los autores.

\section{Literatura citada}

Armendáriz, E.; Jaramillo, F.; Zegarra, L. 2011. Barreras al crecimiento económico de Junín. Diagnóstico y Propuesta 48, Consorcio de Investigación Económica y Social (CIES). Banco Interamericano de Desarrollo.

Arrow, K.; Dasgupta, P.; Goulder, L.; Mumford, K.; Oleson, K. 2012. "Sustainability and the measurement of wealth". Environment and Development Economics 17(3): 317-353.

Barbier, E. 2007. Frontiers and sustainable economic development. Environmental and Resource Economics 37: 271-295. Disponible en: https:// link.springer.com/article/10.1007/ s10640-007-9120-9

BCRP. 2013a. Encuentro económico: Informe económico y social del departamento Junín. Disponible en: http:// www.bcrp.gob.pe/docs/ProyeccionInstitucional/Encuentros-Regionales/2013/junin/ies-junin-2013.pdf

Barco, D.; Vargas, P. 2010. El Perfil del Trabajador Informal y el Retorno de la Educación. Documento de Trabajo 2010-04, Working Paper Series. Banco Central de Reserva del Perú. Disponible en: http://www.bcrp.gob.pe/ docs/Publicaciones/Documentos-deTrabajo/2010/Documento-de-Trabajo-04-2010.pdf

Boza, B. 2006. Canon Minero ¿Caja chica o palanca para el desarrollo? Ciudadanos al Día. Consorcio de Investigación Económica y Social. Disponible en: http://www.cies.org.pe/sites/default/ files/files/otrasinvestigaciones/archivos/canon-minero-caja-chica-opalanca-para-el-desarrollo.pdf

Cantuarias, C.; Orihuela, C. 2010. Testing the Hartwick Rule in the Peruvian
Mining Sector. Universite Montesquieu-Bordeaux IV. Documento no publicado.

Dasgupta, P.; Mäler, K. 2000. Net national product, wealth and social well-being. Environmental and Development Economics 5(1-2):6993. DOI: https://doi.org/10.1017/ $\underline{\mathrm{S} 1355770 \mathrm{X} 00000061}$

Dasgupta, P.; Mäler, K.G. 2001. Wealth as a Criterion for Sustainable Development. World Economics 2(3): 19-44.

Easterly, W.; Levine, R. 2001. It's Not Factor Accumulation: Stylized Facts and Growth Models. World Bank. The World Bank Economic. Review 15(2):177-219. Disponible en: $\underline{\text { https:// }}$ williameasterly.files.wordpress. com $/ 2010 / 08 / 33$ easterly levine itsnotfactoraccumulation prp.pdf

GTZ. 2008. Canon y Regalías: distribución y uso en los Gobiernos Subnacionales. Documento $\mathrm{N}^{\circ} 2$. Publicación Web. Disponible en: http://www.giz-governanceinclusion-social-seguridad.org/wpcontent/uploads/2013/09/Canon-yRegalias 2008.pdf

Hamilton, K.; Atkinson, G. 2006. Wealth, Welfare and Sustainability. Advances in Measuring Sustainable Development. Edward Elgar. Chetelham, UK. 201p.

INEI. 1994. III Censo Nacional Económico 1993. Instituto Nacional de Estadística e Informática. Disponible en: https://www.inei.gob.pe/

INEI. 2008. IV Censo Nacional Económico 2007. Instituto Nacional de Estadística e Informática. Disponible en: $\quad$ http://censos.inei.gob.pe/ cenec2008/redatam inei/

INEI. 2012a. Producto Bruto Interno por Departamentos 2001-2011, año base 1994. Dirección Nacional de Cuentas Nacionales del Perú. Disponible en: http://webapp.inei.gob.pe:8080/ sirtod-series/

INEI. 2012b. Estimaciones y proyecciones de la población económicamente activa, urbana y rural por sexo y grupos de edad, según departamento, 2000 - 
2015. Página: Censo Nacional 1993. Disponible en: http://censos.inei.gob. pe/bcoTablas/CPV93Tablas.htm

INEI. 2012c. Junín: Compendio Estadístico 2011. Oficina Departamental de Estadística e Informática de Junín. Disponible en: http://www. diresajunin.gob.pe/ver documento/ id/0057551e46ba18f196̄c58b27bb3d 5e40557698.pdf/

INEI. 2013a. Cuentas nacionales: producción, consumo intermedio y valor agregado de los sectores extractivos en valores corrientes y reales para el periodo 1994-2011. Documento no publicado.

INEI. 2013b. III Censo Nacional Agropecuario 1994: Disponible en: https://webinei.inei.gob.pe/anda inei/index.php/catalog/566. Fecha de actualización: 25/09/2013.

INEI. 2013c. Compendio Estadístico del Perú 2013: Tomo No 1. Instituto Nacional de Estadística e Informática. Disponible en: https://www.inei. gob.pe/media/MenuRecursivo/ publicaciones digitales/Est/ Lib1097/libro.pdf

INEI y MINAG. 2013. IV Censo Nacional Agropecuario 2012-Resultados Definitivos. Instituto Nacional de Estadística e Informática / Ministerio de Agricultura y Riego. 62 p.

Koopmans, T.C. 1960. "Stationary ordinal utility and impatience". Econométrica (28): 287-309. Disponible en: http:// folk.uio.no/gasheim/zKoo1960.pdf

Koopmans, T.C. 1965. "On the concept of optimal economic growth". Pontificae Academiae Scientiarium Scripta Varia (28): 225-300.

MEF. 2011. Marco Macroeconómico Multi Anual 2012-2014. Ministerio de Economía y Finanzas. Disponible en: $\quad$ http://www.mef.gob.pe/ contenidos/pol econ/marco_macro/ MMM2012_2014.pdf

Ministerio de Energía y Minas. 2013a. Anuario Minero 2012. Disponible en: http://www.minem.gob.pe/ publicacion.php?idSector=1\&idPubl icacion $=450$. Fecha de actualización: 20/10/2013.
Ministerio de Energía y Minas. 2013b. Boletín Estadístico de Minería 2013. Disponible en: http://www. minem.gob.pe/minem/archivos/ file/Mineria/PUBLICACIONES/ VARIABLES/2013/bOLETIN6.pdf. Página 31.

Orihuela, C. 2014. ¿El departamento Junín ha estado en la senda del desarrollo sostenible? Una aplicación basada en el enfoque de sostenibilidad débil. Informe de consultoría presentado al Consorcio de Investigacion Económica y Social (CIES) y Fondo de Estudios y Consultorías BelgaPeruano. Disponible en: http:// www.cies.org.pe/es/investigaciones/ desarrollo-rural-descentralizaciony-modernizacion-del-estado-medioambiente-0

Perroti, D.; Sánchez, R. 2011. La brecha en infraestructura en América Latina y el Caribe. Serie Recursos Naturales e Infraestructura 153. CEPAL. Disponible en: https://www.cepal. org/sites/default/files/events/files/ sanchez_perrotti_2011 brecha infraestructura.pdf

Remy, M.; de los Ríos, C. 2012. Dinámica del mercado de la tierra en América Latina. Concentración y extranjerización y el Caribe: El caso de Perú. Oficina Regional de la FAO para América Latina y el Caribe. Disponible en: http://www.rlc.fao. org/fr/publications-et-documents/ dinamicas-mercado-tierra/

Samuelson, P. 1961. The Evaluation of 'Social Income': Capital Formation and Wealth. En F. A. Lutz and D. C. Hague (eds.), The Theory of Capital. New York: St. Martin's Press. Disponible en: https://link.springer.com/ apter/10.1007/978-1-349-08452-4_3

Seminario, B.; Beltrán, A. 1998. Crecimiento económico en el Perú 1896-1995: Nuevas evidencias estadísticas. Centro de Investigación de la Universidad del Pacífico. Disponible en: $\quad$ http://repositorio.up.edu.pe/ bitstream/handle/11354/1164/DT32. pdf? sequence $=1$

Seminario, B. 2009. Perú: Stock de Capital, 
PIB potencial y Productividad. Disponible en: https://sites.google. com/site/lbseminario/peru-2021

UNU-IHDP y UNEP. 2012. Inclusive Wealth Report 2012. Measuring progress toward sustainability. Cambridge: Cambridge University Press. Disponible en: http://www.ihdp.unu. edu/docs/Publications/Secretariat/ Reports/SDMs/IWR $\% 20$ SDM $\% 20$ Low\%20Resolution.pdf

World Bank. 2006. Where is the Wealth of the Nations?. Measuring Capital for the 21 st Century. Washington DC. USA. Disponible en: https://siteresources. worldbank.org/NTEI/2145781110886258964/20748034/All.pdf

World Bank. 2011. The changing wealth of nations. Washington, DC: World Bank. Disponible en: https:// siteresources.worldbank.org/ ENVIRONMENT/Resources/ ChangingWealthNations.pdf

Yamada, G.; Castro, J. 2010. Educación superior e ingresos laborales: Estimaciones paramétricas y no paramétricas de la rentabilidad por niveles y carreras en el Perú. Documento de Discusión DD/10/06. Universidad del Pacifico. 